\title{
Suplementos nutricionales para prevención de diabetes mellitus gestacional: lecciones aprendidas basadas en la evidencia
}

\author{
Enrique Reyes-Muñoz ${ }^{*}$, Salvador Espino y Sosa², Claudia M. Flores-Robles ${ }^{3}$, \\ Lidia Arce-Sánchez ${ }^{3}$, Nayeli Martínez-Cruz y Pedro Gutiérrez-Castrellón $n^{4,5}$ \\ ${ }^{1}$ Coordinación de Endocrinología Ginecológica y Perinatal, Instituto Nacional de Perinatología Isidro Espinosa de los Reyes; ${ }^{2}$ Subdirección de \\ Investigación clínica, Instituto Nacional de Perinatología Isidro Espinosa de los Reyes; ${ }^{3}$ Coordinación de Endocrinología, Instituto Nacional de \\ Perinatología Isidro Espinosa de los Reyes; ${ }^{4}$ Centro de Investigación Translacional en Ciencias de la Salud, Hospital General Dr. Manuel Gea \\ González; ${ }^{5}$ Sociedad Latinoamericana de Gastroenterologia, Hepatología y Nutrición Pediátrica. Ciudad de México, México
}

\section{Resumen}

Introducción: La diabetes mellitus gestacional (DMG) afecta a entre el 5 y el $40 \%$ de mujeres embarazadas. Recientemente se han evaluado diferentes intervenciones con suplementos nutricionales para prevenir la DMG. Objetivo: Realizar una síntesis de la evidencia sobre eficacia de suplementos nutricionales (mioinositol, probióticos y vitamina D) para prevenir DMG. Método: Se realizó una búsqueda sistemática en PubMed y la biblioteca Cochrane, se incluyeron revisiones sistemáticas de estudios clínicos aleatorizados (ECAs), publicados en idioma inglés o español hasta mayo de 2020; se utilizaron las palabras clave: "prevención", "diabetes gestacional", "hiperglicemia y embarazo", "suplementación", "probióticos", "mio-inositol" y "vitamina D". Resultados: Se analizaron 10 revisiones sistemáticas que cumplieron los criterios de inclusión. La suplementación con mioinositol comparado con placebo disminuyó la incidencia de DMG (RR: 0.44; IC 95\%: 0.27-0.87; cinco ECAs). Si bien la suplementación con vitamina $D$ o probióticos durante el embarazo podría disminuir la incidencia de DMG, la evidencia es limitada. Conclusiones: La suplementación con mioinositol es efectiva para prevenir DMG en mujeres de alto riesgo. La suplementación con vitamina $D$ o probióticos probablemente disminuye la incidencia de DMG.

PALABRAS CLAVE: Diabetes gestacional. Embarazo. Probióticos. Mioinositol. Vitamina D.

\section{Nutritional supplements in the prevention of gestational diabetes mellitus: Evidence- based lessons learned}

\section{Abstract}

Introduction: Gestational diabetes mellitus (GDM) affects between 5 and $40 \%$ of pregnant women. Recently different interventions with nutritional supplements have been evaluated for prevention of GDM. Aim: To perform a synthesis of the evidence on the efficacy of nutritional supplements (myo-inositol, probiotics, and vitamin D) in the prevention of GDM. Methods: A systematic search in PubMed and Cochrane library was performed, including systematic reviews of randomized clinical trials (RCTs), published in English or Spanish until May 2020, using the keywords: "prevention", "gestational diabetes", "hyperglycemia and pregnancy", "supplementation", "probiotics", "myo-inositol" and "vitamin D". Results: 10 systematic reviews that met the inclusion criteria were analyzed. Myo-inositol supplementation compared to placebo decreased the incidence of GDM (RR: 0.44 [0.27-0.87]; five RCTs), low quality of evidence. Although the supplementation with vitamin D

Correspondencia:

*Enrique Reyes-Muñoz

E-mail: dr.enriquereyes@gmail.com
Gac Med Mex. 2020;156(Supl 3): S43-S50

$\begin{array}{lr}\text { Fecha de aceptación: 08-11-2020 } & \text { Disponible en PubMed } \\ \text { DOI: 10.24875/GMM.M20000437 www.gacetamedicademexico.com }\end{array}$

$\begin{array}{lr}\text { Fecha de aceptación: 08-11-2020 } & \text { Disponible en PubMed } \\ \text { DOI: 10.24875/GMM.M20000437 www.gacetamedicademexico.com }\end{array}$ CC BY-NC-ND (http://creativecommons.org/licenses/by-nc-nd/4.0/). 
or probiotics during pregnancy could reduce the incidence of GDM the evidence is limited. Conclusions: Myo-inositol supplementation is effective for prevention of GDM in high-risk women. Supplementation with vitamin D or probiotics probably decreases the incidence of GDM.

KEY WORDS: Gestational diabetes. Pregnancy. Probiotics. Myo-inositol. Vitamin D.

\section{Introducción}

La diabetes mellitus gestacional (DMG) se define como la diabetes que se diagnostica en el segundo o tercer trimestre del embarazo y que claramente no es una diabetes manifiesta ${ }^{1}$. La DMG es la condición metabólica más común durante el embarazo y puede ocasionar complicaciones a corto y largo plazo tanto para la madre como para la descendencia $^{2,3}$. El $15.8 \%$ de los nacidos vivos en el mundo en 2019 padecieron algún tipo de diabetes en el embarazo, de los cuales el $83.6 \%$ se debió a DMG, mientras que el $7.9 \%$ fue el resultado de diabetes detectada antes del embarazo y el $8.5 \%$ por diabetes (incluidos los tipos 1 y 2) detectada por primera vez en el embarazo ${ }^{4}$. En México, la prevalencia nacional de DMG es desconocida, sin embargo, los estudios realizados en mujeres mexicanas han reportado una prevalencia entre el $10 \mathrm{y}$ el $30.1 \%^{5-7}$.

El principal mecanismo fisiopatológico que conduce al desarrollo de DMG es el deterioro de las células $\beta$ en un contexto crónico de resistencia a la insulina en los tejidos de las mujeres durante el embarazo ${ }^{8}$, aunado al incremento en resistencia a la insulina durante la segunda mitad del embarazo, por aumento de hormonas locales y placentarias tales como factor de necrosis tumoral alfa, progesterona, estrógenos, lactógeno placentario, hormona del crecimiento placentaria, cortisol y leptina ${ }^{8}$.

La mayoría de los factores de riesgo para el desarrollo de DMG tienen en común un incremento en la resistencia a la insulina; recientemente se ha propuesto un índice de masa corporal (IMC) $>25 \mathrm{~kg} / \mathrm{m}^{2}$ más uno de los siguientes: sedentarismo, familiar en primer grado con diabetes, grupo étnico de alta prevalencia de diabetes (latino, afroamericano, asiático), antecedente de recién nacido macrosómico, hipertensión o tratamiento antihipertensivo, colesterol vinculado a lipoproteínas de alta densidad $<35 \mathrm{mg} / \mathrm{dl}$, triglicéridos $>250 \mathrm{mg} / \mathrm{dl}$, síndrome de ovarios poliquísticos, hemoglobina glucosilada $\geq 5.7 \%$ o prediabetes, acantosis nigricans y antecedente de enfermedad cardiovascular ${ }^{9,10}$.
Diferentes guías internacionales han recomendado realizar un diagnóstico y tratamiento oportuno de DMG para disminuir el riesgo de complicaciones maternofetales, entre ellas: preeclampsia, cesárea, macrosomía, neonatos grandes para edad gestacional, hipocalcemia e hiperbilirrubinemia neonatal ${ }^{9-12}$. Así como prevenir las complicaciones a largo plazo para la mujer que desarrolló $\mathrm{DMG}^{13}$ y los efectos asociados en los hijos de mujeres con DMG derivados de la programación fetal ${ }^{2,3}$. Sin embargo, existen pocas recomendaciones en relación con la prevención en mujeres con factores de riesgo para desarroIlar DMG.

En el contexto de una epidemia mundial de obesidad, la mayoría de las mujeres embarazadas tienen al menos un factor de riesgo para $\mathrm{DMG}^{9,10}$. Previo al embarazo no existen estrategias de prevención efectivas y el tamaño de la población objetivo es considerable ${ }^{14}$.

Una revisión sistemática Cochrane evaluó los efectos de las intervenciones dietéticas en combinación con ejercicio para prevenir DMG y demostró una posible disminución del riesgo de DMG en el grupo de intervención con dieta y ejercicio en comparación con el grupo de atención estándar (riesgo relativo [RR]: 0.85; intervalo de confianza del 95\% [IC 95\%]: 0.711.01; 6,633 mujeres; 19 estudios clínicos aleatoriza$\operatorname{dos}[\mathrm{ECA}])^{15}$.

En la última década ha habido un creciente interés por evaluar intervenciones preventivas para DMG. Los suplementos nutricionales podrían ser una estrategia segura, asequible y efectiva para prevenir la DMG; entre los suplementos nutricionales que han sido evaluados en ECA se incluyen: mioinositol, probióticos y vitamina $\mathrm{D}^{16}$.

El mioinositol es un nutriente que se encuentra en cereales, leguminosas y carnes, que se utiliza para la formación de la membrana celular y está implicado en la regulación de la vía de señalización de la insulina ${ }^{17,18}$. Diferentes estudios han mostrado que el mioinositol tiene un efecto en la sensibilización a la insulina ${ }^{19,20}$ y por lo tanto una disminución en la resistencia a la insulina. Diferentes autores han demostrado el rol del mioinositol en el embarazo por su 
acción en la reducción de hiperglucemia y la prevención de DMG ${ }^{18,21}$.

Durante el embarazo, un desequilibrio en la flora intestinal favorece una disfunción metabólica con aumento de inflamación, contenido de energía y disminución de la sensibilidad a la insulina ${ }^{22}$. La Organización Mundial de la Salud define los probióticos como «microorganismos vivos que cuando se administran en cantidades adecuadas confieren un beneficio para la salud del huésped» ${ }^{23}$. La suplementación de probióticos disminuye los niveles de marcadores inflamatorios, incluido el factor de necrosis tumoral alfa y la interleucina 6, y aumentan las concentraciones de péptido similar al glucagón tipo 1, incrementando la sensibilidad a la insulina en mujeres embarazadas $^{24}$. Varios ECA han reportado el efecto benéfico de los probióticos sobre el perfil metabólico en la mujer embarazada ${ }^{24}$.

Así mismo, la vitamina $D$ es una vitamina liposoluble que proviene principalmente de la exposición al sol, se encuentra en forma natural en pocos alimentos como aceites de hígado de pescado, champiñones, yemas de huevo e hígado ${ }^{25}$. Entre las diferentes acciones de la vitamina $D$, se ha documentado que regula los niveles de glucemia, mediante la unión y activación del receptor de vitamina $D$ en las células beta pancreáticas, regulando la liberación de insulina en respuesta al nivel de glucosa circulante ${ }^{25,26}$. En algunos ECA se ha reportado un posible efecto beneficioso de la suplementación prenatal con vitamina $D$ en la prevención de $\mathrm{DMG}^{27}$.

El objetivo de esta revisión es proporcionar la evidencia disponible sobre la eficacia de los suplementos nutricionales en la prevención de DMG.

\section{Métodos}

Se realizó una búsqueda sistemática en PubMed y en la biblioteca Cochrane, se utilizaron las palabras clave: "prevención", "diabetes gestacional", "hiperglucemia y embarazo", "mio-inositol", "probióticos" y "vitamina D". Se incluyeron revisiones sistemáticas de $E C A$, publicados en idioma inglés o español hasta mayo de 2020. Se excluyeron revisiones que no utilizaran alguna metodología para evaluar la calidad de la evidencia o que no reportaran el efecto de las intervenciones sobre la incidencia de DMG. Dos investigadores realizaron la búsqueda y seleccionaron los estudios con base en el título y el resumen. Se seleccionaron todos los artículos que potencialmente cumplieron los criterios de inclusión y dos autores revisaron el texto completo de manera individual y evaluaron los criterios de inclusión o exclusión y su calidad metodológica. La extracción de datos y síntesis de la evidencia se realizó recolectando la siguiente información: tipo de estudio, número de ECA incluidos, criterio diagnóstico para DMG, características de los participantes y calidad de la evidencia. La información se sintetizó en tablas de resumen. Se contestaron las siguientes preguntas de investigación clínica: 1) ¿cuál es la eficacia de la suplementación con mioinositol en mujeres de alto riesgo para prevenir DMG comparado con placebo o control?; 2) ¿cuál es la eficacia de la suplementación con probióticos en mujeres de alto riesgo para prevenir DMG comparado con placebo o control?; 3) ¿cuál es la eficacia de la suplementación con vitamina $D$ en mujeres de alto riesgo para prevenir DMG comparado con placebo o control?, y 4) ¿cuál es la eficacia de la suplementación con ácidos grasos omega-3 en mujeres de alto riesgo para prevenir DMG comparado con placebo o control?

\section{Resultados}

Inicialmente se identificaron 340 artículos. Al revisar por título y por suplemento específico se identificaron 15 revisiones para mioinositol, 21 para probióticos y 12 para vitamina $D$, de los cuales se revisó el resumen y se seleccionaron 12 revisiones que se analizaron en extenso, de las cuales dos fueron excluidas. En la biblioteca Cochrane se encontraron 34 revisiones, de las cuales se incluyeron tres, una para cada uno de los suplementos analizados, mismas que se habían identificado por la búsqueda en PubMed.

\section{Mioinositol}

Se analizaron cuatro revisiones sistemáticas ${ }^{28-31}$. Una fue excluida porque no reportó la calidad de la evidencia. En todas las revisiones, con la suplementación con mioinositol comparado con placebo o control hubo una disminución de la incidencia de DMG; la calidad de la evidencia fue baja utilizando la escala GRADE (Tabla 1). En el análisis de sensibilidad hubo homogeneidad cuando se incluyen los cuatro ECA realizados en Italia ( $\left.I^{2}: 0 \% ; p=0.58\right)$, sin embargo, al incluir el estudio realizado en Irlanda ${ }^{32}$ se observa una heterogeneidad significativa ( $\left.1^{2}: 81 \% ; p=0.0002\right)$. La dosis administrada de mioinositol fue de $2 \mathrm{~g}$ dos veces al día en cuatro ECA y $1.1 \mathrm{~g}$ más $27.6 \mathrm{mg}$ de 
Tabla 1. Resultados de revisiones sistemáticas sobre eficacia de la suplementación con mioinositol comparado con placebo para la prevención de diabetes mellitus gestacional (DMG)

\begin{tabular}{|c|c|c|c|}
\hline Estudio & Intervención & $\mathbf{N}$ & Resultados \\
\hline Crawford, et al., $2015^{28}$ & $\begin{array}{l}\text { Suplementación antenatal } \\
\text { con mioinositol vs. } \\
\text { placebo }\end{array}$ & $\begin{array}{l}3 \text { estudios clínicos } \\
\text { aleatorizados; } 502 \\
\text { mujeres }\end{array}$ & $\begin{array}{l}\text { Reducción en la incidencia de DMG. RR: } 0.43 \text { (0.29-0.69). } \\
\text { Calidad de la evidencia: baja (GRADE) }\end{array}$ \\
\hline Guo, et al., $2018^{31}$ & $\begin{array}{l}\text { Suplementación antenatal } \\
\text { con mioinositol vs. } \\
\text { placebo }\end{array}$ & $\begin{array}{l}4 \text { estudios clínicos } \\
\text { aleatorizados; } 586 \\
\text { mujeres }\end{array}$ & $\begin{array}{l}\text { Disminución en la incidencia de DMG. RR: } 0.44 \text { (0.32- } \\
\text { 0.62). Calidad de la evidencia: baja (GRADE) }\end{array}$ \\
\hline Zhang, et al., 201930 & $\begin{array}{l}\text { Suplementación antenatal } \\
\text { con mioinositol vs. } \\
\text { placebo }\end{array}$ & $\begin{array}{l}5 \text { estudios clínicos } \\
\text { aleatorizados; } 927 \\
\text { mujeres }\end{array}$ & $\begin{array}{l}\text { Disminución en la incidencia de DMG. RR: } 0.43 \text { (0.21- } \\
\text { 0.89); Calidad de la evidencia: alta (Jadad scale) } \\
\text { Disminución en la incidencia de parto pretérmino. RR:0.36 } \\
\text { (0.17-0.73). Calidad de la evidencia: alta (Jadad scale) }\end{array}$ \\
\hline Vitagliano, et al., $2019^{29}$ & $\begin{array}{l}\text { Suplementación antenatal } \\
\text { con mioinositol vs. } \\
\text { placebo }\end{array}$ & $\begin{array}{l}4 \text { estudios clínicos } \\
\text { aleatorizados: } 848 \\
\text { mujeres }\end{array}$ & $\begin{array}{l}\text { Disminución en la incidencia de DMG. OR: } 0.49 \text { ( } 0.24- \\
\text { 1.03). Calidad de la evidencia: muy baja (GRADE) } \\
\text { Disminución en la incidencia de parto pretérmino. OR: } 0.35 \\
\text { (0.17-0.74). Calidad de la evidencia: muy baja (GRADE) }\end{array}$ \\
\hline
\end{tabular}

d-chiro-inositol. El inicio de la intervención se realizó entre las 12 y 16 semanas de gestación (SDG) y se incluyeron solo embarazos únicos. El factor de riesgo para inclusión de participantes en dos ECA fue sobrepeso, en un ECA obesidad, en un ECA glucosa de ayuno entre 92 y $125 \mathrm{mg} / \mathrm{dl}$ y en dos ECA el antecedente familiar de diabetes en primer grado. El diagnóstico de DMG se realizó entre las semanas 24-28 utilizando los criterios de la Asociación internacional de grupos de estudio de diabetes y embarazo (IADPSG) ${ }^{33,34}$ en cuatro ECA y en un ECA no se especificó el criterio diagnóstico. En los cinco ECA el objetivo primario fue la prevención de DMG. No hubo efectos secundarios en las mujeres que recibieron mioinositol en ninguno de los ECA.

En una revisión sistemática ${ }^{29}$ se realizó el subanálisis para comparar el efecto con base en la dosis de mioinositol. Utilizando la dosis de $2 \mathrm{~g}$ dos veces al día hubo una disminución significativa en la incidencia de DMG (RR: 0.38; IC 95\%: 0.16-0.87), en cambio al utilizar la dosis de $1.1 \mathrm{~g}$ más $27.6 \mathrm{mg}$ de d-chiro-inositol no hubo un beneficio para prevenir DMG, con una incidencia de DMG del $23 \%$ en el grupo de mioinositol y del $18 \%$ en el grupo placebo (RR: 1.15 ; IC 95\%: 0.87-1.54).

Otro desenlace secundario evaluado fue la incidencia de nacimiento pretérmino, que disminuyó significativamente con la suplementación de mioinositol. No hubo diferencias en desenlaces secundarios como incidencia de cesárea, trastornos hipertensivos, distocia de hombros, desgarros perineales, macrosomía, edad gestacional a la resolución, peso del recién nacido al nacimiento, hipoglucemia neonatal e ingreso a unidad de cuidados intensivos neonatales.

\section{Probióticos}

Se analizaron tres revisiones sistemáticas ${ }^{24,35}$, se excluyó una revisión porque no reportaba el efecto de los probióticos sobre la incidencia de DMG. En dos revisiones se reportó un efecto benéfico en la prevención de DMG, en la revisión más reciente no hubo un beneficio de la suplementación con probióticos para la prevención de DMG (Tabla 2). Solo dos ECA, incluidos las dos últimas revisiones, tuvieron como objetivo primario la incidencia de DMG, en el resto de ECA fue un objetivo secundario. El criterio para el diagnóstico de DMG en la mayoría de los estudios fue el de la IADPSG a las 24-28 SDG. El tipo de probiótico y dosis de administración fue muy heterogéneo entre los diferentes estudios. En la revisión más reciente ${ }^{24,35,36}$, en que se incluyeron siete ECA para el desenlace de DMG, la duración promedio de la intervención desde el inicio de la intervención a la realización de la curva de tolerancia oral a la glucosa fue de 4 a 13 semanas, con un promedio de 9.5 semanas. Si bien la intervención en la mayoría de los estudios se administró hasta el final del embarazo. En cuatro ECA se utilizaron juntos Bifidobacterium Lactis y Lactobacillus Rhamnosus, dosis de 1x109 (dos estudios) y $1 \times 1,010$ (dos estudios) unidades formadoras de colonias (UFC) por cápsula por día, un ECA, 
Tabla 2. Resultados de revisiones sistemáticas sobre eficacia de la suplementación con probióticos comparado con placebo para la prevención de diabetes mellitus gestacional (DMG)

\begin{tabular}{|c|c|c|c|}
\hline Estudio & Intervención & $\mathbf{N}$ & Resultados y calidad de la evidencia \\
\hline $\begin{array}{l}\text { Cochrane } \\
\text { Barret, et al., } 2014^{35}\end{array}$ & $\begin{array}{l}\text { Suplementación antenatal } \\
\text { con probióticos vs. placebo } \\
\text { o control activo }\end{array}$ & $\begin{array}{l}1 \text { estudios clínicos } \\
\text { aleatorizados; } 225 \text { mujeres }\end{array}$ & $\begin{array}{l}\text { Disminución en la incidencia de DMG. RR: } 0.38 \text { (0.20- } \\
0.70) \text {. Calidad de la evidencia: bajo riesgo de sesgos, } \\
\text { evidencia insuficiente }\end{array}$ \\
\hline Han, et al., $2019^{24}$ & $\begin{array}{l}\text { Suplementación antenatal } \\
\text { con probióticos vs. placebo } \\
\text { o control activo }\end{array}$ & $\begin{array}{l}3 \text { estudios clínicos } \\
\text { aleatorizados; } 659 \text { mujeres }\end{array}$ & $\begin{array}{l}\text { Disminución en la incidencia de DMG. RR: } 0.52 \\
(0.34-0.80), p=0.003 \text {. Calidad de la evidencia: bajo } \\
\text { riesgo de sesgos, no especifica calidad }\end{array}$ \\
\hline Masulli, et al., $2020^{36}$ & $\begin{array}{l}\text { Suplementación antenatal } \\
\text { con probióticos vs. placebo } \\
\text { o control activo }\end{array}$ & $\begin{array}{l}7 \text { estudios clínicos } \\
\text { aleatorizados; } 1966 \\
\text { mujeres }\end{array}$ & $\begin{array}{l}\text { No hubo diferencias en la incidencia de DMG. OR: } \\
0.77(0.51,1.16) \text {. Calidad de la evidencia: moderada } \\
\text { (GRADE) }\end{array}$ \\
\hline
\end{tabular}

Tabla 3. Resultados de revisiones sistemáticas sobre eficacia de la suplementación con vitamina D comparado con placebo para la prevención de diabetes mellitus gestacional (DMG)

\begin{tabular}{|c|c|c|c|}
\hline Estudio & Intervención & $\mathrm{N}$ & Resultados y calidad de la evidencia \\
\hline $\begin{array}{l}\text { Pérez-López, } \\
\text { et al., } 2015^{37}\end{array}$ & $\begin{array}{l}\text { Suplementación antenatal con } \\
\text { vitamina D vs. placebo }\end{array}$ & $\begin{array}{l}3 \text { estudios clínicos } \\
\text { aleatorizados; } 384 \text { mujeres }\end{array}$ & $\begin{array}{l}\text { No hubo diferencias en la incidencia de DMG. RR: } 1.05 \\
\text { (0.60-1.84). Calidad de la evidencia: baja (GRADE) }\end{array}$ \\
\hline $\begin{array}{l}\text { Roth, et al., } \\
2017^{38}\end{array}$ & $\begin{array}{l}\text { Suplementación antenatal con } \\
\text { vitamina D vs. placebo o control } \\
\text { activo. }\end{array}$ & $\begin{array}{l}5 \text { estudios clínicos } \\
\text { aleatorizados; } 1,050 \text { mujeres }\end{array}$ & $\begin{array}{l}\text { No hubo diferencias en la incidencia de DMG. RR: } 0.65 \\
\text { (0.39-1.08). Calidad de la evidencia: baja (GRADE) }\end{array}$ \\
\hline \multirow[t]{2}{*}{$\begin{array}{l}\text { Palacios, } \\
\text { et al., } 2019^{27}\end{array}$} & $\begin{array}{l}\text { Suplementación antenatal con } \\
\text { vitamina D vs. placebo }\end{array}$ & $\begin{array}{l}4 \text { estudios clínicos } \\
\text { aleatorizados; } 446 \text { mujeres }\end{array}$ & $\begin{array}{l}\text { Disminución en la incidencia de DMG. RR: } 0.51 \text { (0.27- } \\
\text { 0.97). Calidad de la evidencia: moderada (GRADE) }\end{array}$ \\
\hline & $\begin{array}{l}\text { Suplementación antenatal } \\
\text { con vitamina D más calcio vs. } \\
\text { placebo }\end{array}$ & $\begin{array}{l}1 \text { estudio clínico } \\
\text { aleatorizado; } 54 \text { mujeres }\end{array}$ & $\begin{array}{l}\text { No hubo diferencias en la incidencia de DMG. RR: } 0.33 \\
\text { (0.01-7.84). Calidad de la evidencia: muy baja (GRADE) }\end{array}$ \\
\hline
\end{tabular}

Lactobacillus Acid más B. Lactis, dosis de 1x105 UFC en forma de yogur al día, un ECA Lactobacillus Salivarius cápsulas de $1 \times 109$ por día y un ECA L. Rhamnosus 6x109 UFC por cápsula al día. El análisis de sensibilidad mostró una heterogeneidad significativa entre los estudios ( $\left.\right|^{2}: 62 \% ; p=0.02$ ), probablemente asociado a las diferencias en el tiempo de intervención, dosis y tipo de probióticos. La suplementación con probióticos adicionalmente mostró una reducción estadísticamente significativa en la concentración de glucosa, insulina, colesterol y triglicéridos, sin embargo podría no tener significancia clínica. No hubo efectos secundarios a la administración de probióticos en las participantes de los ECA.

\section{Vitamina $D$}

Se analizaron tres revisiones sistemáticas ${ }^{27,37,38}$. Se excluyó una revisión porque fue actualizada por los mismos autores en 2019 y se incluyó esta última. En dos revisiones se incluyeron ECA que compararon la suplementación de vitamina $D$ vs. placebo o un control activo; no hubo un efecto benéfico de la suplementación con vitamina $D$ en la prevención de DMG con calidad de la evidencia baja. En la revisión Cochrane ${ }^{27,37,38}$ se reportó un beneficio de la suplementación con vitamina $\mathrm{D}$ para disminuir la incidencia de DMG (Tabla 3) con calidad de la evidencia moderada. En dicha revisión se incluyeron cuatro ECA que compararon vitamina $D$ vs. placebo. El inicio de la intervención fue: un ECA antes de las 20 SDG, dos ECA después de las 20 SDG y un ECA no reportó el inicio de la intervención. La dosis de vitamina $D$ fue diferente en los cuatro ECA, en uno se administraron 50,000 UI cada 2 semanas por 10 semanas de las 14-16 a las 24-26 SDG, las participantes incluidas tenían IMC normal y no tenían factores de riesgo para $D M G$, el objetivo primario del estudio fue la incidencia de DMG, se realizó una curva de tolerancia oral a la glucosa (CTOG) $75 \mathrm{~g}$ - 2 h entre las 24 y 26 SDG, el 
criterio diagnóstico para DMG fue glucosa de ayuno $>92 \mathrm{mg} / \mathrm{dl}, 1 \mathrm{~h}>140 \mathrm{mg} / \mathrm{dl}$ y $2 \mathrm{~h}>140 \mathrm{mg} / \mathrm{dl}^{39}$. En el segundo ECA se administraron 400 IU/día de vitamina $D$ (colecalciferol-D3) a partir de las 25 hasta las 34 SDG, no se menciona en qué semana ni el criterio para el diagnóstico de $\mathrm{DMG}^{40}$. En el tercer ECA se midió la concentración de vitamina $D$ y se clasificó como suficiente: $>50 \mathrm{nmol} / /$, insuficiente: $25-50 \mathrm{nmol} / \mathrm{l}$ y deficiente: $<25 \mathrm{nmol} / /$; dependiendo de la concentración de vitamina $\mathrm{D}$ la dosis de suplementación fue una dosis de 60,000 IU de colecalciferol a las 20 SDG en el grupo de suficiencia, dos dosis de 120,000 UI a las 20 y 24 SDG en el grupo de deficiencia y cuatro dosis de 120,000 UI de colecalciferol a las 20,24, 28 y 32 SDG para el grupo de insuficiencia, no se menciona en qué semana se hizo la CTOG, ni los criterios utilizados para el diagnóstico de DMG, solo hubo un caso de DMG por grupo ${ }^{41}$. En el cuarto ECA, el objetivo primario del estudio fue la incidencia de DMG, el grupo de intervención recibió 5,000 UI/semana de vitamina $D$ desde el ingreso hasta las 26 SDG, el diagnóstico de DMG se realizó con una carga de 75 g y se consideró DMG si a las $2 \mathrm{~h}$ el valor de glucosa estaba entre 140 y $200 \mathrm{mg} / \mathrm{dl}^{42}$. Si bien de los cuatro ECA incluidos solo uno mostró un efecto benéfico, en los otros tres no hubo un beneficio de la suplementación con vitamina D en la incidencia de DMG; el análisis de sensibilidad reportado muestra ausencia de heterogeneidad $\left(I^{2}: 0 \% ; p=0.53\right)^{27,37,38}$.

\section{Discusión}

En la presente revisión se muestra que la suplementación con mioinositol $2 \mathrm{~g}$ dos veces al día desde las 12 SDG, en mujeres con riesgo de DMG, es efectiva para prevenir DMG, aun cuando la calidad de la evidencia es baja y se requieren ECA multicéntricos y con mayor número de participantes. La evidencia actual es insuficiente respecto a la suplementación con probióticos y vitamina $D$, si bien podría existir un posible efecto benéfico de estos dos suplementos para la prevención de DMG, debido al tamaño de muestra limitado y que la dosis, tiempo de duración y tipo de intervención son muy heterogéneos entre los ECA incluidos en las revisiones sistemáticas, se requiere diseñar nuevos ECA con estos suplementos cuyo objetivo primario sea la prevención de DMG en mujeres de alto riesgo.

A la fecha no existen recomendaciones estandarizadas en relación con la dosis de suplementación con vitamina $\mathrm{D}$ especialmente en mujeres con concentraciones deficientes.

La dosis de mioinositol de $2 \mathrm{~g}$ dos veces al día mostró un efecto benéfico en la incidencia de DMG, parto pretérmino, disminución en la concentración media de glucosa en los tres valores de la CTOG ayuno, $1 \mathrm{~h}$ y $2 \mathrm{~h}$. Contrario a la dosis de $1.1 \mathrm{~g}$ más $27.6 \mathrm{mg}$ de d-chiro-inositol que no mostró ningún beneficio en dichos descenlaces ${ }^{29}$. Una de las grandes ventajas de utilizar mioinositol y probióticos en la prevención de DMG es la ausencia de efectos adversos para el binomio madre-feto, así como altas tasas de adherencia ${ }^{27,29}$. La seguridad de la suplementación con vitamina $\mathrm{D}$, sobre todo a grandes dosis, debe ser evaluada en futuros estudios en función de muertes maternas, admisión a terapias neonatales, infección neonatal y nacimientos pretérmino antes de las 32 SDG, que no fueron reportados en ninguno de los ECA incluidos en las revisiones sistemáticas ${ }^{27,37,38}$.

Recientemente se ha demostrado que los cambios en el estilo de vida iniciados antes de las 15 SDG, con un control adecuado de la ganancia de peso gestacional, podría disminuir el riesgo de desarrollar $\mathrm{DMG}^{14,15}$. Si bien varios ensayos clínicos sobre intervenciones en el estilo de vida han demostrado reducciones significativas en el riesgo de DMG, muchos más no han mostrado ningún efecto ${ }^{14}$. Además, las mujeres en riesgo parecen reacias a participar en intervenciones durante el embarazo y con frecuencia no pueden adherirse a estas terapias después de la asignación ${ }^{14}$. La implementación y la adherencia a dichas intervenciones son retos que considerar en la mayoría de las mujeres embarazadas en nuestra población. Dentro de las limitaciones de esta revisión podemos mencionar que la búsqueda se limitó a revisiones sistemáticas en dos sistemas de búsqueda y publicaciones en idioma inglés y español. Las fortalezas del estudio son que se incluyeron revisiones que analizaron en forma específica el efecto de los suplementos nutricionales sobre la incidencia de DMG y que se reporta la calidad de la evidencia.

Actualmente el enfoque preventivo en las principales complicaciones del embarazo (entre ellas DMG, preeclampsia, nacimiento pretérmino, hemorragia obstétrica, restricción en el crecimiento intrauterino y macrosomía) debe ser una práctica universal para todo médico que participa en el control prenatal, dado que el beneficio no solo será a corto plazo, sino que tendrá repercusiones en la programación fetal y de esta forma, en la vida adulta de la descendencia ${ }^{2,3,43}$. 
Sin duda, en el futuro también se deben evaluar intervenciones múltiples como la combinación de cambios en el estilo de vida aunados a diferentes suplementos nutricionales que es factible administrar en la práctica diaria en forma simultánea.

\section{Conclusiones}

Con base en la evidencia actual, la suplementación con mioinositol desde el inicio del segundo trimestre del embarazo es efectiva para prevenir DMG. La suplementación con probióticos y vitamina $D$ tiene un posible efecto benéfico en la prevención de DMG.

\section{Financiamiento}

El presente estudio fue financiado por Nestlé México.

\section{Conflicto de intereses}

Enrique Reyes-Muñoz, Pedro Gutiérrez-Castrellón y Salvador Espino y Sosa son speakers e integrantes del Expert Team de Nestlé México. El resto de los autores no tienen conflictos de interés.

\section{Responsabilidades éticas}

Protección de personas y animales. Los autores declaran que para esta investigación no se han realizado experimentos en seres humanos ni en animales.

Confidencialidad de los datos. Los autores declaran que en este artículo no aparecen datos de pacientes.

Derecho a la privacidad y consentimiento infor-

mado. Los autores declaran que en este artículo no aparecen datos de pacientes.

\section{Bibliografía}

1. American Diabetes Association. 2. Classification and diagnosis of diabetes: Standards of Medical Care in Diabetes-2020. Diabetes Care. 2020;43:S14-S31.

2. Franzago M, Fraticelli F, Stuppia L, Vitacolonna E. Nutrigenetics, epigenetics and gestational diabetes: consequences in mother and child. Epigenetics. 2019;14:215-35.

3. Burlina S, Dalfrà MG, Lapolla A. Short- and long-term consequences for offspring exposed to maternal diabetes: a review. J Matern Fetal Neonatal Med. 2019;32:687-94.

4. Diabetes Atlas 2019 [Internet]. International Diabetes Federation [fecha de consulta: 18 de mayo de 2020). Disponible en: https://www.diabetesatlas.org/upload/resources/material/20200302_133351_IDFATLAS9e-final-web.pdf.

5. Ramírez Torres MA. [Gestational diabetes mellitus. Experience at a third level hospital]. Ginecol Obstet Mex. 2005;73:484-91.

6. Reyes-Muñoz E, Parra A, Castillo-Mora A, Ortega-González C. Effect of the diagnostic criteria of the International Association of Diabetes and Pregnancy Study Groups on the prevalence of gestational diabetes mellitus in urban Mexican women: a cross-sectional study. Endocr Pract. 2012;18:146-51.
7. Dainelli L, Prieto-Patron A, Silva-Zolezzi I, Sosa-Rubi SG, Espino y Sosa S, Reyes-Muñoz E, et al. Screening and management of gestational diabetes in Mexico: results from a survey of multilocation, multi-health care institution practitioners. Diabetes Metab Syndr Obes. 2018;11:105-16.

8. Agha-Jaffar R, Oliver N, Johnston D, Robinson S. Gestational diabetes mellitus: does an effective prevention strategy exist? Nat Rev Endocrinol. 2016;12:533-46.

9. Committee on Practice Bulletins-Obstetrics. ACOG Practice Bulletin, Number 190. Gestational diabetes mellitus. Obstet Gynecol. 2018; 131:e49-e64.

10. Webber J, Charlton M, Johns N. Diabetes in pregnancy: management of diabetes and its complications from preconception to the postnatal period (NG3). Br J Diabetes. 2015;15:107.

11. HAPO Study Cooperative Research Group, Metzger BE, Lowe LP, Dyer AR, Trimble ER, Chaovarindr U, et al. Hyperglycemia and adverse pregnancy outcomes. N Engl J Med. 2008;358:1991-2002.

12. American College of Obstetricians and Gynecologists. Committee on Practice Bulletins-Obstetrics. Macrosomia: ACOG Practice Bulletin, Number 216. Obstet Gynecol. 2020;135:e18-e35

13. Association AD, American Diabetes Association. 3. Prevention or delay of type 2 diabetes: Standards of Medical Care in Diabetes-2020. Diabetes Care. 2020;43:S32-S36.

14. Egan AM, Simmons D. Lessons learned from lifestyle prevention trials in gestational diabetes mellitus. Diabet Med. 2019;36:142-50.

15. Shepherd E, Gomersall JC, Tieu J, Han S, Crowther CA, Middleton P. Combined diet and exercise interventions for preventing gestational diabetes mellitus. Cochrane Database Syst Rev. 2017 Nov 13;11(11): CD010443.

16. Plows JF, Reynolds CM, Vickers MH, Baker PN, Stanley JL. Nutritional supplementation for the prevention and/or treatment of gestational diabetes mellitus. Curr Diab Rep. 2019;19:73.

17. Croze ML, Soulage CO. Potential role and therapeutic interests of myo-inositol in metabolic diseases. Biochimie. 2013;95:1811-27.

18. Reyes-Muñoz E, Guardo FD, Ciebiera M, Kahramanoglu I, Sathyapalan T, Lin L-T, et al. Diet and nutritional interventions with the special role of myo-inositol in gestational diabetes mellitus management. An Evidence-Based Critical Appraisal. Curr Pharm Des. 2019;25:2467-73.

19. Corrado F, D'Anna R, Di Vieste G, Giordano D, Pintaudi B, Santamaria A, et al. The effect of myoinositol supplementation on insulin resistance in patients with gestational diabetes. Diabet Med. 2011;28:972-5.

20. Croze ML, Géloën A, Soulage CO. Abnormalities in myo-inositol metabolism associated with type 2 diabetes in mice fed a high-fat diet: benefits of a dietary myo-inositol supplementation. Br J Nutr. 2015:113:1862-75.

21. Santamaria A, Alibrandi A, Di Benedetto A, Pintaudi B, Corrado F, Facchinetti $F$, et al. Clinical and metabolic outcomes in pregnant women at risk for gestational diabetes mellitus supplemented with myo-inositol: a secondary analysis from 3 RCTs. Am J Obstet Gynecol. 2018;219:300. e1-300.e6.

22. DiGiulio DB, Callahan BJ, McMurdie PJ, Costello EK, Lyell DJ, Robaczewska $A$, et al. Temporal and spatial variation of the human microbiota during pregnancy. Proc Natl Acad Sci U S A. 2015;112:11060-5.

23. Report of the Joint FAO/WHO Expert Consultation on Evaluation of Health and Nutritional Properties of Probiotics in Food Including Powder Milk with Live Lactic Acid Bacteria [Internet]. Food and Agriculture Organization of the United Nations, World Health Organization; 2002. Disponible en: http://www.fao.org/documents/pub dett.asp?lang=en\&pub id=61756

24. Han M-M, Sun J-F, Su X-H, Peng Y-F, Goyal H, Wu C-H et al. Probiotics improve glucose and lipid metabolism in pregnant women: a meta-analysis. Ann Transl Med. 2019;7:99.

25. Holick MF. Vitamin D status: measurement, interpretation, and clinical application. Ann Epidemiol. 2009;19:73-8.

26. Clifton-Bligh RJ, McElduff $P$, McElduff A. Maternal vitamin D deficiency, ethnicity and gestational diabetes. Diabet Med. 2008;25:678-84.

27. Palacios C, Kostiuk LK, Peña-Rosas JP. Vitamin D supplementation for women during pregnancy. Cochrane Database Syst Rev. 2019;7:CD008873.

28. Crawford TJ, Crowther CA, Alsweiler J, Brown J. Antenatal dietary supplementation with myo-inositol in women during pregnancy for preventing gestational diabetes. Cochrane Database Syst Rev. 2015;2015(12): CD011507.

29. Vitagliano A, Saccone G, Cosmi E, Visentin S, Dessole F, Ambrosini G, et al. Inositol for the prevention of gestational diabetes: a systematic review and meta-analysis of randomized controlled trials. Arch Gynecol Obstet. 2019;299:55-68.

30. Zhang H, Lv Y, Li Z, Sun L, Guo W. The efficacy of myo-inositol supplementation to prevent gestational diabetes onset: a meta-analysis of randomized controlled trials. J Matern Fetal Neonatal Med. 2019;32:2249-55.

31. Guo X, Guo S, Miao Z, Li Z, Zhang H. Myo-inositol lowers the risk of developing gestational diabetic mellitus in pregnancies: A systematic review and meta-analysis of randomized controlled trials with trial sequential analysis. J Diabetes Complications. 2018;32:342-8.

32. Farren M, Daly N, McKeating A, Kinsley B, Turner MJ, Daly S. The prevention of gestational diabetes mellitus with antenatal oral inositol supplementation: A randomized controlled trial. Diabetes Care. 2017;40:759-63. 
Gaceta Médica de México. 2020;156(Supl 3)

33. Weinert LS. International Association of Diabetes and Pregnancy Study Groups recommendations on the diagnosis and classification of hyperglycemia in pregnancy: comment to the International Association of Diabetes and Pregnancy Study Groups Consensus Panel. Diabetes Care. 2010;33:e97; author reply e98.

34. International Association of Diabetes and Pregnancy Study Groups Consensus Panel, Metzger BE, Gabbe SG, Persson B, Buchanan TA, Catalano PA, et al. International association of diabetes and pregnancy study groups recommendations on the diagnosis and classification of hyperglycemia in pregnancy. Diabetes Care. 2010;33:676-82.

35. Barrett HL, Dekker Nitert M, Conwell LS, Callaway LK. Probiotics for preventing gestational diabetes. Cochrane Database Syst Rev. 2014;2014(2):CD009951.

36. Masulli M, Vitacolonna E, Fraticelli F, Della Pepa G, Mannucci E, Mona$\mathrm{mi}$ M. Effects of probiotic supplementation during pregnancy on metabolic outcomes: A systematic review and meta-analysis of randomized controlled trials. Diabetes Res Clin Pract. 2020;162:108111.

37. Pérez-López FR, Pasupuleti V, Mezones-Holguin E, Benites-Zapata VA, Thota $P$, Deshpande A, et al. Effect of vitamin D supplementation during pregnancy on maternal and neonatal outcomes: a systematic review and meta-analysis of randomized controlled trials. Fertil Steril. 2015;103:1278-88.e4.
38. Roth DE, Leung M, Mesfin E, Qamar H, Watterworth J, Papp E. Vitamin D supplementation during pregnancy: state of the evidence from a systematic review of randomised trials. BMJ. 2017;359:j5237.

39. Tehrani HG, Mostajeran F, Banihashemi B. Effect of vitamin D supplementation on the incidence of gestational diabetes. Adv Biomed Res. 2017;6:79.

40. Asemi Z, Samimi M, Tabassi Z, Shakeri H, Esmaillzadeh A. Vitamin D supplementation affects serum high-sensitivity C-reactive protein, insulin resistance, and biomarkers of oxidative stress in pregnant women. J Nutr. 2013:143:1432-8

41. Sablok A, Batra A, Thariani K, Batra A, Bharti R, Aggarwal AR, et al. Supplementation of vitamin $D$ in pregnancy and its correlation with feto-maternal outcome. Clin Endocrinol. 2015;83:536-41.

42. Shahgheibi S, Farhadifar F, Pouya B. The effect of vitamin D supplementation on gestational diabetes in high-risk women: Results from a randomized placebo-controlled trial. J Res Med Sci. 2016;21:2.

43. Eriksson JG. Developmental origins of health and disease - from a small body size at birth to epigenetics. Ann Med. 2016:48:456-67. 